\title{
Development of a pyramidal trap for monitoring fruit-piercing stink bugs baited with Plautia crossota stali (Hemiptera: Pentatomidae) aggregation pheromone $^{\dagger}$
}

\author{
Ishizue ADACHI, ${ }^{1 *}$ Ken UCHINO ${ }^{2}$ and Fumiaki MoCHIZUKI ${ }^{3}$ \\ ${ }^{1}$ National Institute of Fruit Tree Science, National Agriculture and Food Research Organization; Tsukuba, Ibaraki 305-8605, Japan \\ ${ }^{2}$ Chiba Prefectural Agriculture Experiment Station; Togane, Chiba 283-0804, Japan \\ ${ }^{3}$ Specialty Chemicals Research Center, Shin-Etsu Chemical Co., Ltd.; Joetsu, Niigata 942-8601, Japan
}

(Received 11 January 2007; Accepted 16 April 2007)

\begin{abstract}
Field studies were conducted to evaluate the effectiveness of an improved pyramidal trap for monitoring fruit-piercing stink bugs such as Plautia crossota stali Scott, Halyomorpha halys (Stål), and Glaucias subpunctatus (Walker) in two regions with different stink bug population densities. Improved traps, made from either yellow or translucent and colorless materials, were compared with the original pyramidal trap and the standard water-basin trap. All traps were baited with $P$. c. stali aggregation pheromone. The translucent, colorless trap captured significantly more target insects than the original design or the yellow trap, although there was no significant difference in one region. Further, the translucent improved design trap caught stink bugs comparable to the water-basin trap, and fluctuations in capture of P. c. stali were proportionally similar between the two trap types. The improved trap design eliminates the need for changing water in the standard water-basin trap, thus the improved trap may be used as an effective and simple monitoring tool over a wide geographical range.
\end{abstract}

Key words: Traps; monitoring; stink bugs; fruit; pheromone

\section{INTRODUCTION}

Forty-five species of fruit-piercing stink bugs have been reported in Japan (Japanese Society of Applied Entomology and Zoology, 2006). Of these, three pentatomid species, the brown-winged green stink bug Plautia crossota stali Scott, the brown marmorated stink bug Halyomorpha halys (Stål), and Glaucias subpunctatus (Walker) are especially critical because they are abundant and cause severe fruit damage. $P$. c. stali is distributed throughout most of Japan. H. halys and G. subpunctatus tend to be predominant in cool and warm regions, respectively. Stink bug populations erupted in 1973 over a wide area of Japan (Hasegawa and Umeya, 1974). Periodic outbreaks have since occurred, causing severe damage to fruit trees such as citrus (Ide, 1997), apple (Funayama, 1996), stone fruits, fig (Watanabe, 1996; Sato, 1997), persimmon
(Yanase, 1997), and loquat (Katase et al., 2005).

Because stink bugs are polyphagous, and the adults are highly mobile, they invade from woodlands or uncultivated areas into fruit orchards, requiring frequent insecticide applications to prevent infestations. However, integrated pest management (IPM) strategies use narrow-spectrum chemicals to replace broad-spectrum insecticides, allowing pesticide dosage and/or frequency of applications to be reduced. In such a narrow-spectrum, reducedspray environment, it is likely that stink bugs will become an increasing menace. Apple, pear and peach are crops in which mating disruption techniques using synthetic sex pheromones against key Lepidopteran pests have largely replaced insecticides. Stink bugs have indeed emerged as a major threat to these crops.

To effectively manage stink bugs, it is extremely important to monitor their abundance or activity.

\footnotetext{
* To whom correspondence should be addressed at: E-mail: adachii@affrc.go.jp

${ }^{\dagger}$ Contribution No. 1457 of the National Institute of Fruit Tree Science, NARO

DOI: $10.1303 /$ aez.2007.425
} 
Light traps have been used (e.g., Moriya et al., 1987; Katayama et al., 1993) for monitoring stink bugs, but they also attract other nocturnal insects. In addition, it is difficult to install light traps in many places, including woodlands, because of the difficulty of providing an electric power supply. The ability to monitor stink bugs was greatly improved by the identification of $P$. c . stali aggregation pheromone, methyl (E,E,Z)-2,4,6-decatrienoate (PcsAP) (Sugie et al., 1996). As a result of this advance, a water-basin trap baited with PcsAP has become an effective tool for estimating the density of stink bugs (e.g. Nakamura and Nishino, 1999). However, the water-basin trap requires frequent replenishment and replacement of water, which restricts the area of installation. We have thus developed a new type of pheromonebaited waterless trap (Shimizu and Adachi, 2004; Katase et al., 2005). This "dry", pyramidal-shaped trap made it possible to continuously monitor stink bugs, although the number of catches was often less than in water-basin traps. Subsequently, the structure of the original pyramidal trap has been changed to improve trapping effectiveness. The purpose of this study was to assess the adult stink bug capture efficiencies of variations on the improved pyramidal trap.

\section{MATERIALS AND METHODS}

The experiments were carried out in Togane, on 4.2 ha at the Chiba Prefectural Agriculture Experiment Station, Chiba Prefecture, and in Tsukuba, on 17.2 ha at the National Institute of Fruit Tree Science, Ibaraki Prefecture, Japan. Several kinds of deciduous fruit trees were planted among the windbreaks of coniferous trees such as Japanese cypress, Chamaecyparis obtusa, and Japanese cedar, Cryptomeria japonica, in both regions. Windbreak plantings were adjacent to open fields, weedy areas, vegetable fields and greenhouses.

Four trap types were used. The standard waterbasin trap was the yellow type of Kogane-call (Sankei Chemical Co., Japan) with a 3.0-liter basin (referred to as Trap C, hereafter; Fig. 1C). The original pyramidal trap was constructed of 5-mmthick plastic danplate (Nichidai Industry Co., Japan) and a 2-liter transparent polyethylene terephthalate (PET) bottle (Trap A; Fig. 1A; Katase et al., 2005). The improved pyramidal trap was composed of three parts: landing board, induction funnel, and collection container (Figs. 1B and 2). The former two parts were made from polypropylene (PP), and the latter acrylonitrile butadiene styrene (ABS). The landing board was constructed of four panels, each of which was ca. $29 \mathrm{~cm}$ in height, $27 \mathrm{~cm}$ in width at the base, and $6 \mathrm{~cm}$ in width at the top. A slit was cut $8 \mathrm{~cm}$ in length by $4 \mathrm{~cm}$ in width in the center of the landing board. The induction funnel was $16 \mathrm{~cm}$ in width at the base and $3 \mathrm{~cm}$ in width at the top with a 4-cm-long chimney-like projection. The collection container
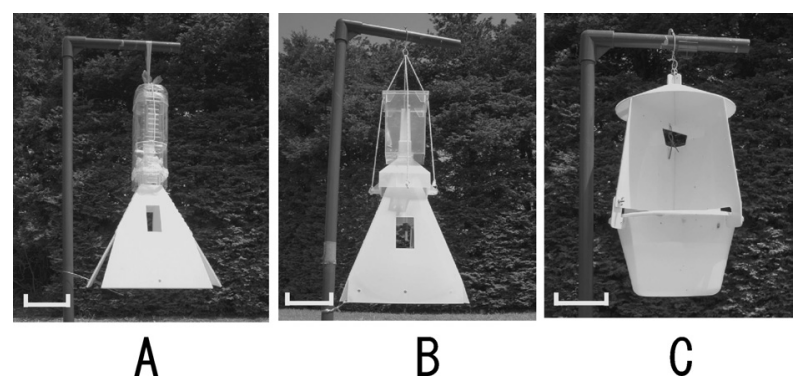

Fig. 1. Original pyramidal trap (A), improved pyramidal trap (B), and water-basin trap (C) used for stink bug monitoring. Scale bars in the figures indicate $10 \mathrm{~cm}$.

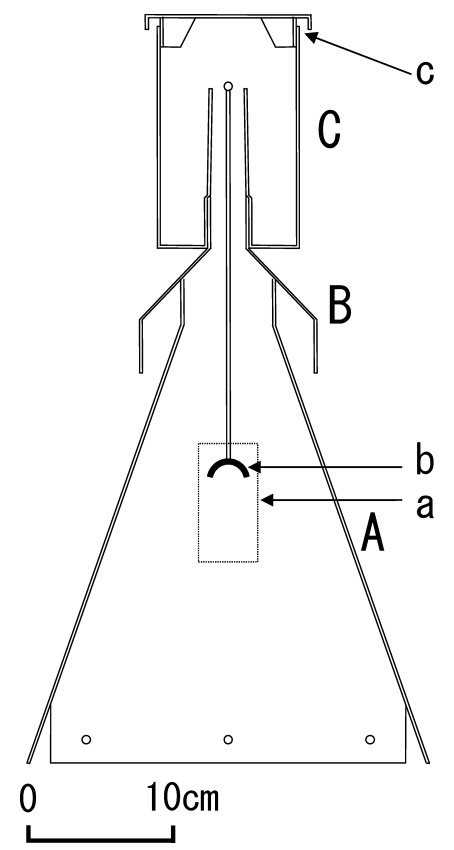

Fig. 2. Profile of improved pyramidal trap. Landing board (A), induction funnel (B), and collection container (C). A $8 \times 4-\mathrm{cm}$-long slit cut in the center of the landing board (a), a lure of the Plautia crossota stali aggregation pheromone attached at the end of the stick (b), and the opening under the lid of the collection container for ventilation (c). 
was ca. 10 (width $) \times 10$ (depth) $\times 15$ (height) $\mathrm{cm}$, and was set by attaching it to the induction funnel. The opening under the lid of the collection container allowed for ventilation. Because the insects need to walk from the landing board to the collection container through the induction funnel, thin polypropylene sheets with an average roughness $(R a)$ of $3.2 \mu \mathrm{m}$ were overlaid on the passage surface. A $25-\mathrm{cm}$-long stick was hung in the center of the trap to provide another path on which stink bugs could ascend toward the collection container. A lure of PcsAP was attached at the tail end of the stick. In the improved pyramidal trap, two color types were prepared. In one, the landing board and the induction funnel were dark yellow (Trap BY) and the other was translucent and colorless (Trap BT). The collection container was transparent in both types. A scrap of DDVP plate (Kokusaieisei Co., Japan) containing $1.0 \mathrm{~g}$ of dichlorvos was added inside the collection containers of all types of pyramidal traps.

Four replicates of three treatments (Trap BY, Trap BT, and Trap C) were established at each test site, and two replicates of Trap A were added at Togane. Traps were baited with one lure containing about $34 \mathrm{mg}$ of PcsAP (Shin-Etsu Chemical Co., Japan), which was replaced every 20 days. All traps were suspended at head height. Within each replicate, traps were rotated clockwise on each trap-check date in Togane, and every 3 days in Tsukuba, to minimize the influence of trapping position. The distance between replicates was more than $200 \mathrm{~m}$, and the distance between traps within replicates was ca. $20 \mathrm{~m}$.

Traps were checked for captured stink bugs at one- to three-day intervals from September 1 until November 15 in 2005 in Togane and from July 21 until November 15 in 2005 in Tsukuba. Thus, a total of 56 and 61 trap-checks were carried out in Togane and Tsukuba, respectively.

Trap capture data were accumulated across every 5 days to minimize periodic variation, and analyzed using the statistical package JMP for Windows (SAS Institute, 2002). Before data analysis, counts were transformed using $\log (x+1)$ to satisfy the homogeneity of variances and standardization of means. Actual means and standard errors of the mean (SEM) are presented in the text, tables and figures. When the analysis of variance (ANOVA) indicated significant differences, multi- ple comparisons of the means were performed using the Tukey-Kramer honestly significant difference (HSD) test at $p=0.05$ (Sokal and Rohlf, 1995). Finally, to evaluate the "synchronization" (Athanassiou et al., 2004) between pairs of catches among the different trap types on the same date, linearity of regression was tested and the correlation coefficients were tested for departure from zero at $p=0.01$ using the JMP statistical software.

\section{RESULTS}

The total numbers of adult stink bugs caught in each trap type during the entire experimental period are presented in Table 1. Trapping period data from the two regions extended over nearly 11 weeks in Togane and nearly 17 weeks in Tsukuba, and the number of replicates differed between the regions or among trap types. There were approximately ten times more $P$. c. stali adult captures in Togane than in Tsukuba. H. halys and G. subpunctatus captures were also higher in Togane than in Tsukuba, but the differences were small compared to $P$. c. stali. Thus, the experiments were carried out under conditions of different stink bug population densities in the two regions.

Sex ratios of $P$. c. stali adults caught by each trap type were significantly female-biased, except for the BY traps in Tsukuba (Table 1). There was no significant difference between the sex ratios of BT and $\mathrm{C}$ traps in either Togane $\left(\chi^{2}=2.15 ; p=0.142\right)$ or Tsukuba $\left(\chi^{2}=0.07 ; p=0.789\right)$. H. halys and $G$. subpunctatus sex ratios varied little by place or trap type.

The interaction of trap type $\times$ location was not significant in either region (Table 2), and consequently there was no effect of location on trap type. Significant differences were recorded for $P$. $c$. stali trapping from both regions among all three trap types (Table 2). Significantly more adults were captured in Trap C than in Trap BY (Fig. 3). Trap BT also caught more adults than Trap BY, although the difference between them was not significant at Togane. No significant differences were detected between Trap BT and Trap C in either region. There were also significant differences in G. subpunctatus trapping among the three trap types both at Togane $(F=4.62 ; \quad \mathrm{df}=2,177 ; \quad p=0.011)$ and Tsukuba $(F=7.80 ; \mathrm{df}=2,285 ; p<0.001)$. The numbers of adults caught with Traps BT and C were not statis- 
Table 1. Total numbers and sex ratios of captured Plautia crossota stali, Halyomorpha halys and Glaucias subpunctatus adults for each trap type

\begin{tabular}{|c|c|c|c|c|c|c|c|c|c|c|c|c|}
\hline \multirow{2}{*}{$\begin{array}{l}\text { Region } \\
\text { Trap type }\end{array}$} & \multicolumn{4}{|c|}{ P. c. stali } & \multicolumn{4}{|c|}{ H. halys } & \multicolumn{4}{|c|}{ G. subpunctatus } \\
\hline & Male & Female & Total & q ratio & Male & Female & Total & q ratio & Male & Female & Total & q ratio \\
\hline \multicolumn{13}{|l|}{ Togane $\mathrm{a}^{\mathrm{a}}$} \\
\hline Trap A & 245 & 472 & 717 & $0.66^{* * *}$ & 16 & 10 & 26 & 0.38 & 32 & 34 & 66 & 0.52 \\
\hline Trap BY & 385 & 710 & 1,095 & $0.65^{* * *}$ & 36 & 41 & 77 & 0.53 & 67 & 73 & 140 & 0.52 \\
\hline Trap BT & 971 & 1,460 & 2,431 & $0.60 * * *$ & 71 & 90 & 161 & 0.56 & 237 & 253 & 490 & 0.52 \\
\hline Trap C & 1,960 & 2,735 & 4,695 & $0.58 * * *$ & 30 & 41 & 71 & 0.58 & 294 & 364 & 658 & $0.55^{* *}$ \\
\hline \multicolumn{13}{|c|}{ Tsukuba $^{\mathrm{b}}$} \\
\hline Trap BY & 27 & 40 & 67 & 0.60 & 15 & 5 & 20 & $0.25 *$ & 16 & 21 & 37 & 0.57 \\
\hline Trap BT & 115 & 152 & 267 & $0.57 *$ & 21 & 12 & 33 & 0.36 & 78 & 65 & 143 & 0.45 \\
\hline Trap C & 204 & 281 & 485 & $0.58 * * *$ & 19 & 17 & 36 & 0.47 & 111 & 119 & 230 & 0.52 \\
\hline
\end{tabular}

Trap A, original pyramidal trap; Trap BY, yellow improved pyramidal trap; Trap BT, translucent, colorless improved pyramidal trap; Trap C, water-basin trap.

${ }^{a}$ Traps were checked from September 1 to November 15 in 2005. Four replicates were performed for Trap BY, Trap BT, and Trap C, and two replicates for Trap A.

${ }^{\mathrm{b}}$ The traps were checked from July 21 to November 15 in 2005 . Four replicates were performed for each trap type.

${ }^{\mathrm{c}}$ Female/(Female + Male). Asterisks indicate sex ratios significantly different from $1: 1$ ( $\chi^{2}$-test). Levels of significance: $* p<0.05, * * p<0.01, * * * p<0.001$.

Table 2. ANOVA statistics for main effects (trap type and trapping location) and their interaction on the catches of Plautia crossota stali (total $\mathrm{df}=179$ in Togane, and 287 in Tsukuba)

\begin{tabular}{lrrr}
\hline \multicolumn{1}{c}{ Effects } & df & $F$ & \multicolumn{1}{c}{$p$} \\
\hline Togane & & & \\
$\quad$ Trap type & & & \\
$\quad$ Location & 3 & 3.233 & 0.0419 \\
$\quad$ Trap type $\times$ Location & 6 & 0.527 & 0.6642 \\
Tsukuba & & & 0.8308 \\
$\quad$ Trap type & 2 & 14.432 & $<0.0001$ \\
$\quad$ Location & 3 & 8.879 & $<0.0001$ \\
Trap type $\times$ Location & 6 & 4.332 & 0.4155 \\
\hline
\end{tabular}

${ }^{\text {a }}$ Three trap types (BY, BT, and C) were examined.

tically different, but were significantly greater than Trap BY in both regions. H. halys trapping did not significantly differ among trap types at either Togane $(F=1.92 ; \mathrm{df}=2,177 ; p=0.150)$ or Tsukuba $(F=0.83 ; \mathrm{df}=2,285 ; p=0.436)$.

P. c. stali captures varied considerably during the trapping period (Fig. 4). In Togane, about $57 \%$ of the adults were captured in BY traps in September, with another $43 \%$ in early October, whereas BT and $\mathrm{C}$ traps accounted for ca. $70 \%$ and $30 \%$ during the two peak trapping periods. There were also two peak trapping periods in Tsukuba. About $73 \%$ of captures in BY traps were in mid-September, with

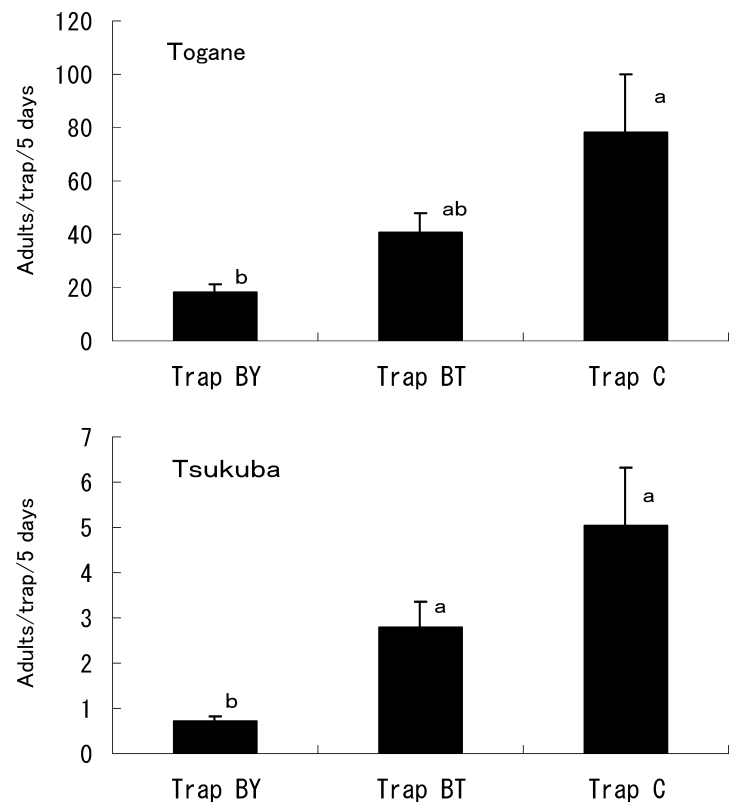

Fig. 3. Mean \pm SEM numbers of Plautia crossota stali adults captured in each trap type per 5 days in Togane and Tsukuba. Bars with the same letter are not significantly different (Tukey-Kramer HSD test at $p=0.05$ ).

$27 \%$ in early October. BT and C traps had peaks of about $85 \%$ and $15 \%$. Trap C contained significantly more $P$. c. stali adults than the other two trap types on some trap-check dates during the trapping peaks (Fig. 4), largely because Trap C caught ex- 

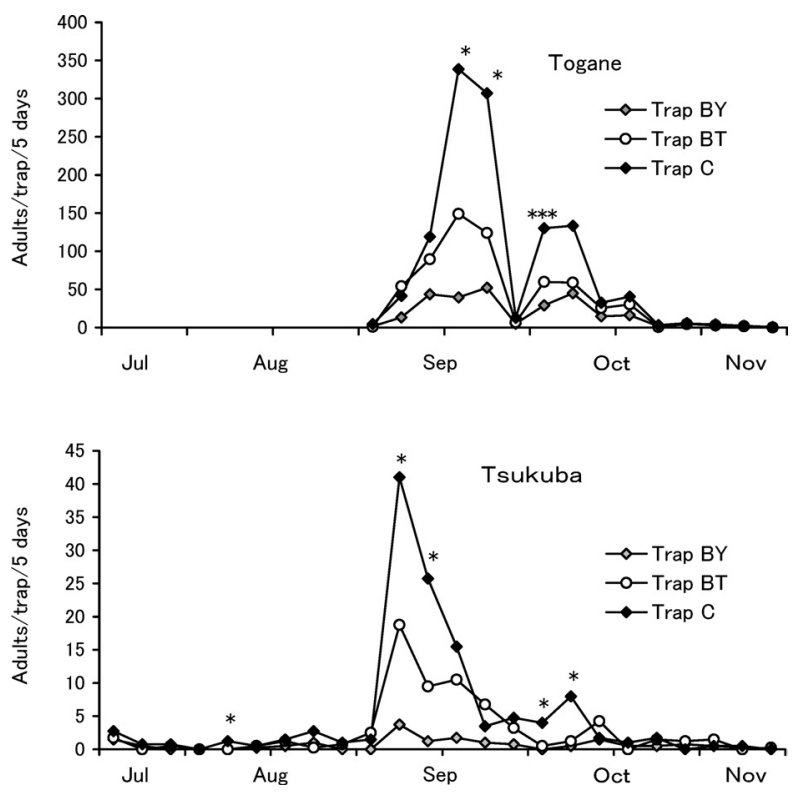

Fig. 4. Mean numbers of captured Plautia crossota stali adults for each trap type per 5 days during the experimental period in Togane and Tsukuba. An ANOVA was performed separately for each date to determine on which dates the differences among trap types were significant at the $0.05\left(^{*}\right)$, or $0.001(* * *)$ levels. Dates with no asterisk indicate that there were no significant differences.

Table 3. Detection sensitivity in terms of the number of Plautia crossota stali adults captured within a day for Trap BT and Trap C at Togane

\begin{tabular}{clc}
\hline \multirow{2}{*}{$\begin{array}{c}\text { No. of adults } \\
\text { captured by } \\
\text { each trap }\end{array}$} & \multicolumn{2}{c}{ No. of traps with each trap type } \\
\cline { 2 - 3 } & Trap BT & Trap C \\
\hline 0 & $51(33.6 \%)$ & $31(20.4 \%)$ \\
$1-10$ & $59(38.8)$ & $71(46.7)$ \\
$11-100$ & $39(25.7)$ & $42(27.6)$ \\
$101-200$ & $3(2.0)$ & $5(3.3)$ \\
$>200$ & 0 & $3^{\mathrm{a}}(2.0)$ \\
\hline
\end{tabular}

a 259 adults were captured on September 14, 518 on September 24, and 844 on September 20.

tremely large numbers of adults even within a day (Table 3).

Correlation coefficients representing the synchronization of captures among the different types of traps were positive and significant for $P$. c. stali (in Togane, $r=0.889$, Trap BY-Trap BT; $r=0.830$, Trap BY-Trap C; and $r=0.896$, Trap BT-Trap C, and in Tsukuba, $r=0.493$, Trap BY-Trap BT; $r=0.541$, Trap BY-Trap C; and $r=0.719$, Trap BT-Trap C). Values were also positive and signifi-

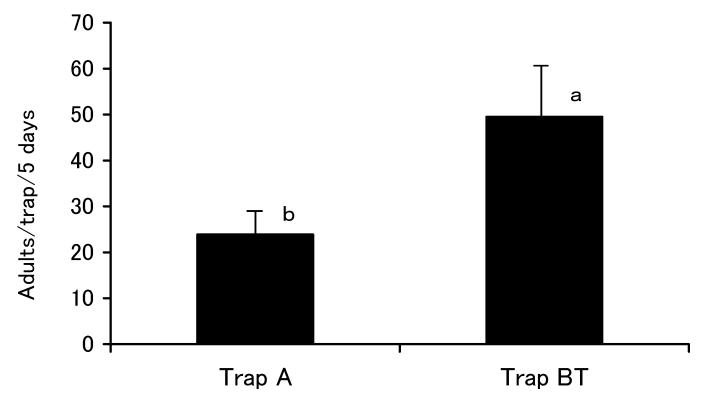

Fig. 5. Mean \pm SEM numbers of Plautia crossota stali adults captured in each trap type per 5 days at Togane. Different letters indicate significant differences (Tukey-Kramer HSD test at $p=0.05)$.

cant for $H$. halys and G. subpunctatus.

The difference in P. c. stali adult capture by Trap A and Trap BT in two replicates at Togane was marginally significant $(F=4.07 ; \mathrm{df}=1,58 ; p=$ $0.048)$ with average ( \pm SEM) catches of $23.9 \pm 4.7$ and 49.5 \pm 11.8 , respectively (Fig. 5).

\section{DISCUSSION}

Similar trapping performance results were obtained from both the Togane and Tsukuba regions for each trap type, irrespective of the difference in stink bug population densities. Trap C (water-basin trap) was the most effective trap for P. c. stali. Trap BY (yellow improved pyramidal trap) was least effective. Trap BT (translucent, colorless improved pyramidal trap) trapping efficacy approached that of Trap C. There was, in fact, no significant difference in captures between Traps BT and C (Fig. 3). However, the almost twofold number of captures in Trap $\mathrm{C}$ at both regions ( 78.3 to 40.5 at Togane and 5.1 to 2.8 at Tsukuba) indicates the high sensitivity of the trap C type. On the other hand, the traps had consistently proportional capturing patterns (Fig. 4), implying that insect populations can be adequately monitored using pyramidal traps as long as populations do not fall below a certain threshold, where the most efficient traps (i.e. Trap C) would be most likely to detect the first indications of a population increase. Therefore, BT traps should be sensitive enough to detect imminent $P$. c. stali population increases as well as to monitor population spikes and decreases, without the inherent drawbacks of the more efficient but labor-intensive water basin-type standard traps.

The total number of adults captured was greater 
in Trap C than in Trap BT, though there were no significant differences detected with the statistical measures used. This difference may be attributed to the trapping mechanism rather than to attraction. The majority of adults attracted to Trap $\mathrm{C}$ drown in soapy water, whereas some portion of the adults attracted to Trap BT escape before reaching the collection container. Thus, it is probable that the trapping efficiency (numbers captured vs. numbers attracted; Athanassiou et al., 2002) differs between trap types. The attractants in terms of the quantity of pheromone or the combination with light can be re-examined, as should the structure and materials of the BT traps to increase the number of adults that are attracted and retained.

Trap A (the original pyramidal trap) captured only half as many P. c. stali adults as Trap BT (Fig. $5)$. One of the major factors causing this discrepancy could be the roughness of the trap surface. Although its roughness was not measured, Trap A was apparently smoother than Trap BT, which possibly allowed more adults to escape from the A traps. Little is known about the optimum surface roughness for trap design. Further trapping experiments using materials with various surface roughnesses can and should be performed. In addition, the Trap BT collection container is easily removable from Trap BT, but is fixed in Trap A (Katase et al., 2005), so that only about $30 \mathrm{~s}$ are required for collecting the captured insects, which is about a quarter of the time required for Trap A (unpublished data).

A large number of phytophagous insects respond positively to yellow (Prokopy and Owens, 1983). Stink bugs such as Euschistus tristigmus were captured in greater numbers in traps coated with yellow than in traps coated with other colors (Leskey and Hogmire, 2005). In the current study, BY traps (yellow improved pyramidal trap) captured significantly fewer $P$. c. stali adults than BT traps (Fig. 3). However, this does not indicate that $P$. c. stali adults do not detect and move to yellow. Many adults were observed to be attracted to Trap BY, but they remained under the yellow landing boards without ascending to the collection container, even in the daytime. In general, fruit-piercing stink bugs stay in the shade, usually provided by the underside of leaves in the daytime. Thus, the yellow-painted landing boards may have obscured their normal behavior of ascending upward.
The current study was carried out from July to November. However, fruit-piercing stink bugs appear starting in late April. Pyramidal trap performance must therefore also be checked in spring and early summer. The current study also revealed that $H$. halys and G. subpunctatus are captured equally in BT and C traps. Although it is unclear why the two species are attracted by the $P$. c. stali aggregation pheromone, Trap BT can likely be useful to monitor their activities.

The fruit-piercing stink bugs, especially $P . c$. stali adults, move through a wide range of habitats, including woodlands and fruit orchards. Accordingly, it is desirable to distribute monitoring traps over a wide geographical area. Water-basin traps have proven to be the most effective type, but have the serious drawback of requiring frequent exchanges of water to maintain trap efficacy. In contrast, the pyramidal trap developed in the current study requires far less attention. Development and optimization of a wide-range trapping system using pyramidal traps would be a useful tool for providing information on stink bug populations and movement for the timing of control measures.

\section{ACKNOWLEDGEMENTS}

This work was supported in part by a Grant-in-Aid for Research Project for Utilizing Advanced Technologies in Agriculture, Forestry and Fisheries (1648) from the Ministry of Agriculture, Forestry and Fisheries, Japan.

\section{REFERENCES}

Athanassiou, C. G., N. G. Kavallieratos, F. T. Gravanis, N. A. Koukounitsas and D. E. Roussou (2002) Influence of trap type, pheromone quantity and trapping location on capture of the pink bollworm, Pectinophora gossypiella (Saunders) (Lepidoptera: Gelechiidae). Appl. Entomol. Zool. 37: 385-391.

Athanassiou, C. G., N. G. Kavallieratos and B. E. Mazomenos (2004) Effect of trap type, trap color, trapping location, and pheromone dispenser on captures of male Palpita unionalis (Lepidoptera: Pyralidae). J. Econ. Entomol. 97: 321-329.

Funayama, K. (1996) Sucking injury on apple fruit by the adult of brown marmorated stink bug Halyomorpha mista (Uhler). Annu. Rep. Plant Prot. North Japan 47: 140-142 (in Japanese with English summary).

Hasegawa, H. and K. Umeya (1974) Outbreak of stink bugs in fruit trees. Plant Prot. 28: 279-286 (in Japanese).

Ide, Y. (1997) Damage of citrus by the outbreak of the stink bugs attacking fruit trees in 1996. Plant Protect. 51: 155-157 (in Japanese).

Japanese Society of Applied Entomology and Zoology (2006) Major Insect and Other Pests of Economic Plants in 
Japan (Revised ed.). Japanese Plant Protection Association, Tokyo. 387 pp.

Katase, M., K. Shimizu, H. Nagasaki and I. Adachi (2005) Application of synthetic aggregation pheromone of Plautia crossota stali Scott to the monitoring and mass trapping of fruit-piercing stink bugs. Bull. Chiba Agric. Res. Cent. 4: 135-144 (in Japanese with English summary).

Katayama, E., T. Fukuda and H. Nozawa (1993) Light trap monitoring of the stink bugs attacking fruit trees and their ovarian development. Bull. Tochigi Agr. Exp. Stn. 40: 59-74 (in Japanese with English summary).

Leskey, T. C. and H. W. Hogmire (2005) Monitoring stink bugs (Hemiptera: Pentatomidae) in mid-Atlantic apple and peach orchards. J. Econ. Entomol. 98: 143-153.

Moriya, S., M. Shiga and M. Mabuchi (1987) Analysis of light trap records in four major species of fruit-piercing stink bugs, with special reference of body size variation in trapped adults of Plautia stali Scott. Bull. Fruit Tree Res. Stn. A 14: 79-94 (in Japanese with English summary).

Nakamura, Y. and T. Nishino (1999) Forecasting fruit damage caused by the brown-winged green bug, Plautia stali Scott, and its occurrence using synthetic aggregation pheromone traps. Kyushu Pl. Prot. Res. 45: 119-122 (in Japanese).

Prokopy, R. J. and E. D. Owens (1983) Visual detection of plants by herbivorous insects. Annu. Rev. Entomol. 28: $337-364$.

SAS Institute (2002) Version 5.0.1a. SAS Institute Inc., Cary, NC.

Sato, R. (1997) Outbreak of the brownwinged green stink bug and its damage to pear in 1996. Plant Prot. 51: 158-159 (in Japanese).

Shimizu, K. and I. Adachi (2004) Dry trap for fruit-piercing stink bugs. Patent Number 3541217. The Japan Patent Office.

Sokal, R. R. and F. J. Rohlf (1995) Biometry. 3rd ed. Freedman, New York. 887 pp.

Sugie, H., M. Yoshida, K. Kawasaki, H. Noguchi, S. Moriya, K. Takagi, H. Fukuda, A. Fujiie, M. Yamanaka, Y. Ohira, T. Tsutsumi, K. Tsuda, K. Fukumoto, M. Yamashita and H. Suzuki (1996) Identification of the aggregation pheromone of the brown-winged green bug, Plautia stali Scott (Heteroptera: Pentatomidae). Appl. Entomol. Zool. 31: 427-431.

Watanabe, K. (1996) Characteristic of damages of Lygocoris (Apolygus) lucorum (Meyer-Dür) (Heteroptera: Miridae) and Halyomorpha halys (Stål) (Heteroptera: Pentatomidae) on cherry. Ann. Rept. Plant Prot. North Japan 47: 143-144 (in Japanese).

Yanase, N. (1997) An analysis of damage caused by extraordinary outbreak of fruit stink bugs in 1996-persimmon-. Plant Prot. 51: 160-162 (in Japanese). 